\title{
An ontology based agile C2 organization model
}

\author{
Tingting Yang ${ }^{1, a}$, Baoxin Xiu ${ }^{1, b}$, Weiming Zhang ${ }^{1, c}$ and Jie Liu ${ }^{1, d}$ \\ Science and technology on Information Systems Engineering Laboratory, National University of \\ Defense Technology, ChangSha, HuNan, China \\ a392387247@qq.com, bmuyi927@gmail.com, '1694498011@qq.com, turtle_muyi927@163.com
}

Keywords: Agility, agile C2 organization, force organizations

\begin{abstract}
Agility is arguably one of the most important characteristics of successful Information Age organizations. The study of agility organizations is currently one of the hottest topics in the organization study field. In this paper, we put forward an agile command and control (C2) organization model and force organization choosing method based on the model and theory of ontology. Base on the model and choosing method, we can automatically and quickly find out the proper force organizations for a coming mission to support the agility of the $\mathrm{C} 2$ organization.
\end{abstract}

\section{Introduction}

Agility is arguably one of the most important characteristics of successful Information Age organizations [1]. With a wider use of information and network technology in the modern social life, new organizational concepts and patterns are coming out, such as network organization, virtual enterprise, agile manufacturing system, spirituality organization and hyperlinks organization tissue $[1,4]$ and so on. These new type organization concepts and patterns broke through the traditional geographical restrictions, organizational resource limitations and restrictions on traditional structure. They present the characteristics of distribution, flattening, flexibility and autonomy of organizations.

Meantime, in the C2 filed, the uncertainty and asymmetry of the battlefield environment lead to the increase of the complexity of the military activity. New types of organization patterns such as the network centric warfare, the "Power to the Edge" [1] and other new type organization pattern appear.

"Power to the Edge" pattern means to organize all the resources and opportunities to present the best power. To cope with the complexity and the uncertainty of the external environment it constantly changes and evolves the organizational structure and behavior. We name the $\mathrm{C} 2$ organization, which can implement the "Power to the Edge" pattern, agile C2 organization.

The study of agile C2 organization is to enhance the robustness and flexibility in the battlefield space, improve effectiveness of platform and get the best position in a combat. It will have important theoretical significances and application values.

In this paper, based on the agile C2 organization and ontology theory, we will build an agile C2 organization ontology model and propose a method to dynamically generate a force organization set for a coming mission. The model and method will be validated by a case of a navy joint coordination landing combat.

\section{Literature review}

Scholars have done a lot of work on research of the organization. In the early 1990s, Kathleen proposed a theory named Computational and Mathematical Organization found (CMOT) [5]. The basic idea of this theory is the essence properties of human organization can also be calculated. This theory adopts the method of computational mathematics to study human organization (also called natural organization) in the form of computing entities.

The Computational Analysis of Social and Organizational Systems (CASOS) center and Institute for Complex Engineered Systems (so-called ICES) of Carnegie - Mellon University used CMOT theory in the military field. They attempt to describe and analyze the forces organization and provide a basis for the construction of forces organization. They proposed the organization model of PCANS 
[6], the forces organization topology model based on PCANS [7] and the model of adaptive organizational behavior based on Simulated Annealing algorithm [8, 9]. These models are mainly for the description and analysis of the organization and its behavior. They are not able to guide the organization of the design effectively.

"Power to the Edge" is firstly proposed by Alberts and Hayesin in 2003. This new kind of organization mode has the characteristics of two aspects. One is to achieve interoperability; the second is to achieve agility. Later in 2011, they propose a set of experiment s based on their ELICIT to find out the way to measure the agility. But still no clear agile C2 organization model has been proposed. Using system engineering technology to design a task orientation organization is the main method to implement the series A2C2 experiments designed by the American army. University of Connecticut and Aptima Company have made great work on its theory, method and technology. But still not reflects the nature of the agile C2 organization.

U.S. navy information age war expert Jeffrey r. Cares has analyzed the research of the characteristic and the insufficiency of current network centric warfare model and puts forward a information age combat model [11] based on the complex network theory. Anthony h. Dekker et al. from Australia's Defense, Science and Technology Organization (DSTO) introduce social network analysis method to the C2 organization research field based on the idea CMOT theory. They establish a new model to descript networked organizational combat named FINC (Force, Intelligence, Networking and C2) [12]. The current researches of network theory mainly focus on the organization description and the analysis of the characteristics of the network. Less work has been done on how to construct the organization.

To sum up, the research on agile C2 organization is mostly embodied in the aspect of theoretical research and no clear model or method has been proposed to realize the agile C2 organization. We are aiming at propose an agile C2 organization model and force organization choosing model based on the agile C2 organization and the ontology theory.Ontology theory is a widely used tool in building models these days. Based on the otology models the computer can do some logical inference itself. Grunner's ontology construction methodology [2, 3] proposed a model using ontology.

In this paper, we will describe our agile C2 organization model using the language of ontology and propose a force organization choosing method to help the model building.

This paper is structured as follows: Section 1 is an introduction, Section 2 briefly introduced the related research, Section 3 is the definition of the basic concepts in the agile C2 organization, Section 4 are the model and choosing method, Section 5 is the experiments and analysis, and Section 6 is conclusion.

\section{Ontology based agile C2 organization model basic concepts}

Basic concepts. The basic concepts of the agile C2 organization include organization, mission, task, organization for mission (OFM), organization for task (OFT), decision-maker, platform, resource and function. All the concepts and their relationships can be seen in the figure 1 below.

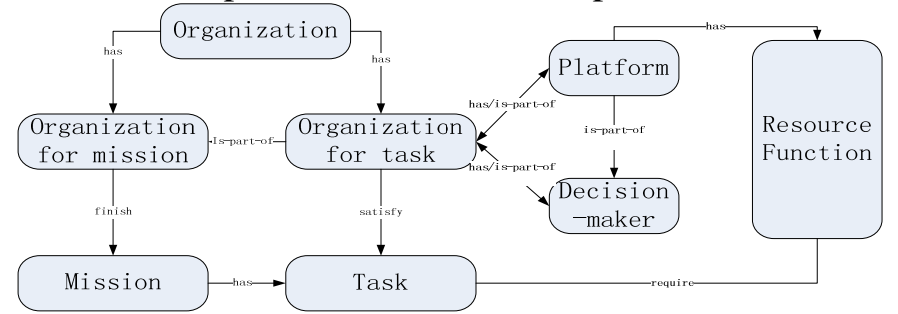

Fig. 1. Basic concepts

Mission describes the combats an agile C2 organization need to take part in. Mission is made up of a collection of tasks and the relationship between the tasks. Mission space is a set of missions. All missions should be put in the space. Each mission has a list of tasks. Building a mission apace with task lists is the most different part compared with the traditional C2 organization.

Task is the basic sub process of a mission. Every task has a requirement of a collection of resources. A task cannot be completed until all the required resources have been provided. 
Agile C2 force organizations can be divided into mission oriented force organization and task oriented force organization when performing a mission and task. The two force organization are respectively called OFM and OFT for short later.OFM consist of a collection of OFTs. Each OFT in can complete at least one task of the mission. OFT consist of a collection of decision-makers, platforms with resources and their structural relationships.

Force organization space is a set too. The space should contain all the OMFs and OFTs. The force organization space has a relationship with the mission space. Each force organization in the force organization space matches with a task of a mission in the mission space. When a new mission comes and the agile $\mathrm{C} 2$ organization has to generate new force organization to complete the mission, the force organization space will get new element too.

Decision-maker has the ability to communicate with other decision-makers and directly connect to a collection of platforms. Decision-maker has the duty to conduct the platforms to complete tasks or missions.

Platform has a collection of resources. Each kind of resources has a special function. All of the functions of the resources belong to the platform together make up the ability of the platform.

The relationships of the elements are has relationship, part_of relationship, satisfied relationship, require relationship and finished relationship.

Has relationship is described with a binary predicate has(x, y). Meaning element $\mathrm{x}$ has element $\mathrm{y}$. Part_of relationship is described with a binary predicate part_of(x, y). Meaning element $\mathrm{x}$ is part of element y. Satisfied relationship is described with a binary predicate satisfied (x, y). Meaning element $\mathrm{x}$ can satisfy the requirements of element $\mathrm{y}$. Require relationship is described with a binary predicate require (x,y). Meaning element $\mathrm{x}$ requires element $\mathrm{y}$. Finished relationship is described by predicate finished(x). Meaning mission x can be finished by the agile C2 organization.These relationships will be discussed detailly later in the next part.

Ontology based agile C2 organization model. The ontology based agile C2 organization model contains all the basic concepts we mentioned above and the relationships between the basic concepts. Definition 1. Mission and task are defined as :

$$
\mathrm{M}(\mathrm{x})::=\{\mathrm{T}(\mathrm{x})\}
$$

We use predicate $\mathrm{M}(\mathrm{x})$ to describe mission and $\mathrm{T}(\mathrm{x})$ to describe task. Each mission can be decomposed to at least one task. They have the relationship of has. Each task requires at least one kind of resource. They have the relationship of part_of.

Definition 2. Decision-maker is defined as:

$$
\mathrm{D}(\mathrm{x})::=<\text { linked decision-maker, linked platform }>
$$

We use predicate $\mathrm{D}(\mathrm{x})$ to describe decision-maker. Each decision-maker has at least one platform. A platform must belong to one and only one decision-maker. Decision-maker and platform have the relationship of has. Platform and decision-maker have the relationship of part-of.

Definition 3. Platform and resources functions are defined as:

$$
\mathrm{P}(\mathrm{x})::=<\text { linked decision-maker, linked } \mathrm{F}(\mathrm{x})>
$$

We use predicate $\mathrm{P}(\mathrm{x})$ to describe platform. Each platform has at least one kind of resources. A resource must belong to one and only one platform. Platform and resource function have the relationship of has. Resource function and platform have the relationship of part-of.

Definition 4. OFM and OFT are defined as:

$$
\operatorname{OFM}(\mathrm{x})::=\{\operatorname{OFT}(\mathrm{x})\}
$$

We use predicate OFM(x) to describe OFM. We use predicate OFT(x) to describe OFT.

Each force organization has at least one decision-maker. They has the relationship of has. The decision-makers in one same OFT must be able to communicate with each other. A platform can be seen as a part of an OFT only when the decision-maker it belongs to is a part of the OFT. A resource can be seen as a part of an OFT only when the platform it belongs to is a part of the OFT. They have the relationship of part-of.

Definition 5. Agile C2 organization is defined as:

Agile C2 Organization::= <organization structure, force organization space, mission space, matching relationship $>$ 
Organization structure::=<decision-maker, platform, resources function >

Force organization space:= $\{$ OFM, OFT $\}$

Mission space::= mission, task list $\}$

Matching relationship::= $($ (OFM, mission), (OFT, task) $\}$

A task can be satisfied by a OFT only when all the resources it requires is provided the OFT. They have the relationship of satisfied. The match is recorded as (OFM, mission).

A mission can be finished by the agile C2 organization only when all the tasks of the mission have been satisfied. They have the relationship of finished. The match is recorded as (OFT, task).

Matching relationship records all the proper match of (OFM, mission) and (OFT, task).

The Matching relationship set grows with the mission space and force organization space. When a new mission come we can search the set first to find exist matches. This will shorted the calculation time if we have the proper match in the set. Therefore, the bigger the set and the spaces are the agiler the C2 organization. So next we will propose a method to help the organization find and update the sets and spaces.

\section{Ontology based force organization choosing method}

According to the basic concepts of the agile C2 organization, the agile C2 organization model and the basic axioms of ontology, we build and the force organization choosing method.

Force organization choosing methods should solve several key problems. First, what are the missions' requirements? Second, what abilities and resources do the OFMs and OFTs have? Third, what kind of situation can be seen as the tasks can be satisfied by OFTs? Forth, what kind of situation can be seen as the missions can be completed by the agile $\mathrm{C} 2$ organization.

Based on model and problems above, we set up the logical inference rules.

First, analysis the missions' and tasks' requirements;

$$
\begin{gathered}
\forall x(M(x) \rightarrow \exists y(T(y) \wedge \operatorname{has}(x, y))) \\
\forall x(T(x) \rightarrow \exists y(F(y) \wedge \text { require }(x, y)))
\end{gathered}
$$

Second, analysis the abilities and resources of the OFMs and OFTs

$$
\begin{array}{r}
\forall x(O M T(x) \rightarrow \exists y(O F T(y) \wedge \text { part_of }(y, x))) \\
\forall x(O F T(x) \rightarrow \exists y(F(y) \wedge \text { part_of }(y, x)))
\end{array}
$$

Third, match the tasks and OFTs?

$$
\forall x \exists y((T(x) \wedge O F T(y) \wedge \text { satisfied }(x, y)) \rightarrow \forall z(F(z) \wedge \text { require }(x, z) \wedge \text { part_of }(z, y)))
$$

Forth, match the missions and the OFMs.

$$
\forall x \exists y((M(x) \wedge O F M(y) \wedge \text { satisfied }(x)) \rightarrow \forall z \exists s(T(z) \wedge O F T(s) \wedge h a s(x, z) \wedge \text { part_of }(s, y)))
$$

Fifth, repeat step one to four until all proper OFTs and OFMs are found. Then we record the matches. The more matches the agile $\mathrm{C} 2$ organization has the faster it will take action when a mission comes.

\section{Case study}

The method will be validated by a case of a navy joint coordination landing combat. Detailed information of the case scenario is showed in the figure and chart bellow.

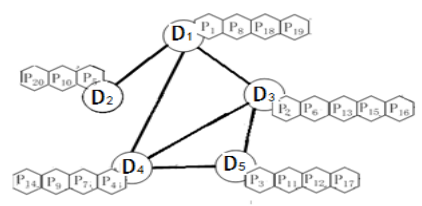

Fig. 2. Decision-makers and platforms

Figure 2 shows the decision-makers, their platforms and the relationships between them. It shows platform 1, 8, 18, 19 belongs to decision-maker 1 and decision-maker 1 can cooperate with decision-maker 2 and 3. 
Table 1 Platforms and resource functions

\begin{tabular}{|l|l|l|l|l|l|l|l|l|}
\hline \multirow{2}{*}{ Platform } & \multicolumn{7}{|c|}{ Resource Function } \\
\cline { 2 - 10 } & $\mathbf{F}_{\mathbf{1}}$ & $\mathbf{F}_{\mathbf{2}}$ & $\mathbf{F}_{\mathbf{3}}$ & $\mathbf{F}_{\mathbf{4}}$ & $\mathbf{F}_{\mathbf{5}}$ & $\mathbf{F}_{\mathbf{6}}$ & $\mathbf{F}_{\mathbf{7}}$ & $\mathbf{F}_{\mathbf{8}}$ \\
\hline 1:DDG & 1 & 1 & 1 & 0 & 1 & 1 & 0 & 0 \\
\hline 2:FFG & 1 & 1 & 1 & 0 & 1 & 1 & 0 & 0 \\
\hline 3:CG & 1 & 1 & 1 & 0 & 1 & 1 & 0 & 0 \\
\hline 4:ENG & 0 & 0 & 0 & 1 & 0 & 0 & & 0 \\
\hline 5:INFA & 1 & 0 & 0 & 1 & 1 & 1 & 1 & 0 \\
\hline 6:SD & 1 & 0 & 0 & 0 & 0 & 0 & 0 & 0 \\
\hline 7:AHI & 1 & 1 & 0 & 0 & 1 & 1 & 1 & 0 \\
\hline$\ldots$ & $\cdots$ & $\cdots$ & $\cdots$ & $\cdots$ & $\cdots$ & $\cdots$ & $\cdots$ & $\cdots$ \\
\hline
\end{tabular}

Table 1 shows the platforms and their resource functions. 1s mean the platform has the resource function while 0s mean does not have.

Table 2 Tasks and resource functions

\begin{tabular}{|l|l|l|l|l|l|l|l|l|}
\hline \multirow{2}{*}{ Task } & \multicolumn{7}{|c|}{ Task Requirement } \\
\cline { 2 - 10 } & $\mathbf{F}_{\mathbf{1}}$ & $\mathbf{F}_{\mathbf{2}}$ & $\mathbf{F}_{\mathbf{3}}$ & $\mathbf{F}_{\mathbf{4}}$ & $\mathbf{F}_{\mathbf{5}}$ & $\mathbf{F}_{\mathbf{6}}$ & $\mathbf{F}_{\mathbf{7}}$ & $\mathbf{F}_{\mathbf{8}}$ \\
\hline $\mathbf{T}_{\mathbf{1}}$ & 1 & 1 & 1 & 0 & 0 & 1 & 0 & 1 \\
\hline $\mathbf{T}_{\mathbf{2}}$ & 1 & 1 & 1 & 0 & 0 & 1 & 0 & 1 \\
\hline $\mathbf{T}_{\mathbf{3}}$ & 0 & 1 & 0 & 0 & 0 & 0 & 0 & 0 \\
\hline $\mathbf{T}_{\mathbf{4}}$ & 0 & 1 & 0 & 0 & 0 & 0 & 0 & 0 \\
\hline $\mathbf{T}_{\mathbf{5}}$ & 0 & 1 & 0 & 0 & 0 & 0 & 1 & 0 \\
\hline $\mathbf{T}_{\mathbf{6}}$ & 0 & 0 & 0 & 1 & 1 & 1 & 0 & 0 \\
\hline $\mathbf{T}_{\mathbf{7}}$ & 0 & 0 & 0 & 1 & 1 & 1 & 0 & 0 \\
\hline$\ldots$ & $\ldots$ & $\ldots$ & $\ldots$ & $\cdots$ & $\ldots$ & $\ldots$ & $\ldots$ & $\cdots$ \\
\hline
\end{tabular}

Table 2 shows the tasks and their requirements of the resource functions. 1s mean the task requires the resource function while 0 s mean does not require.

Take mission $M=\left\{T_{1}, T_{3}, T_{6}\right\}$ as an example.

Then the required resource functions are:

$$
\begin{aligned}
T_{1}= & \left\{F_{1}, F_{2}, F_{3}, F_{6}, F_{8}\right\} \\
T_{3}= & \left\{F_{2}\right\} \quad T_{6}=\left\{F_{4}, F_{5}, F_{6}\right\} \\
& D_{1}=\left\{P_{1}, P_{8}, P_{18}, P_{19}\right\}
\end{aligned}
$$

According to figure 2 the decision-makers and platforms are:

$$
\begin{aligned}
& D_{2}=\left\{P_{5}, P_{10}, P_{20}\right\} \\
& D_{3}=\left\{P_{2}, P_{6}, P_{13}, P_{15}, P_{16}\right\} \\
& D_{4}=\left\{P_{4}, P_{7}, P_{9}, P_{14}\right\} \\
& D_{5}=\left\{P_{3}, P_{11}, P_{12}, P_{17}\right\}
\end{aligned}
$$

First, we start from the first task $T_{1}$ and search from $D_{1}$ 。

Then we get the resources as follows:

$$
\begin{aligned}
& P_{1}=\left\{F_{1}, F_{2}, F_{3}, F_{5}, F_{6}\right\} \quad P_{18}=\left\{F_{1}, F_{4}, F_{5}, F_{6}, F_{7}\right\} \\
& P_{8}=\left\{F_{1}, F_{2}, F_{5}, F_{6}, F_{7}\right\} \quad P_{19}=\left\{F_{1}, F_{4}, F_{5}, F_{6}, F_{7}\right\}
\end{aligned}
$$

According to those constraints we can get a force organization with resources as follow:

$$
\left\{F_{1}, F_{2}, F_{3}, F_{4}, F_{5}, F_{6}, F_{7}\right\}
$$

According to the rules, $F_{8}$ is still needed. So $D_{2}$ is considered. Repeat the procedure to find all OFTs and OFMs.
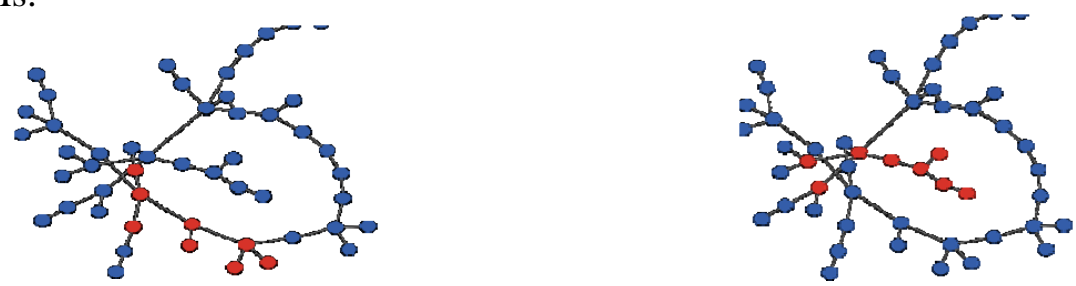

Fig. 3. OFM I and OFM II

The result of the case is a set of OFMs. Two proper OFMs are contained in the set. Figure 3 shows two of the OFMs in the set (the red organizations). The red notes are the decision-makers and their 
platforms. The links are the relationships between them. All the nodes and relationship compose to a proper OFM. Based on this set, we can later do some detailed work to measure the efficiency of the force organizations. Then, we can find a most satisfied OFM with less calculation time too.

\section{Conclusions}

Case above is a simple case with small size of data set. In a real combat problem bigger organization and more complex missions will be involved.

The mission space and force organization space are the most important part in the model. The sizes of the spaces determine the agility of the organizations. The bigger the sizes are the faster the organization will deal with the coming missions as large numbers of matches of the mission and the force organization will have been recorded, which means less calculate time will be needed

Based on this model, later in the future we will build a select method to find the best force organization for a coming mission.

\section{References}

[1] S. Alberts and R. E. Hayes. Power to the Edge: Command Control in the information Age, Information Age Transformation Series, CCRP Publications, 2003.

[2] Uschold II. King etc. The enterprise Ontology. The Knowledge Engineering Review, 1998.13(1): 31-89

[3] Mike Uschold, Michale Grunniger. Ontologies: principles, methods and applications. The Knowledge Engineering Review, 1996.11.

[4] Xiu Baoxin.Design Methodology of C2 Organizational Structure and Its Analysis of Robustness and Adaptivity.2006

[5] Kathleen M. Carley and M. J. Prietula, Computational Organization Theory. Lawrence Erlbaum Associates. Hillsdale. NJ, 1994.

[6] Kathleen M. Carley. Organizational Adaptation. Annals of Operations Research. 1998, 75: 25-47.

[7] Kathleen M Carley, Svoboda, D M. Modeling Organizational Adaptation as a simulated annealing process. Sociological Methods and Research, 1996, 25(1).

[8] Kathleen M Carley, Krackhardt D. A PCANS model of structure in organization. International Symposium on Command and Control Research and Technology, Monterray, CA. 1998.

[9] Kathleen M. Carley and Krackhardt, D. A typology for C2 measures. In: 1999 International Symposium on Command and Control Research and Technology, Newport, RI. 1999.

[10]David S. Alberts. The Agility Advantage A Survival Guide for Complex Enterprises and Endeavors, Monterey, , Sep. 2011,

[11] Jeffrey R. Cares. An Information Age Combat Model 2004.12.30.

[12] Dekker. A. H. Network Topology and Military Performance. Defense Science and Technology Organization, Australia 2005. 